\title{
Momentum of vortex tangles by weighted area information
}

\author{
Simone Zuccher* \\ Department of Computer Science, University of Verona, Cà Vignal 2, Strada Le Grazie 15, 37134 Verona, Italy \\ Renzo L. Ricca ${ }^{\dagger}$ \\ Department of Mathematics and Applications, University of Milano-Bicocca, Via Cozzi 55, 20125 Milano, Italy \\ and BDIC, Beijing University of Technology, 100 Pingleyuan, Beijing 100124, People's Republic of China
}

(Received 3 February 2019; published 15 July 2019)

\begin{abstract}
Here we show how to apply a recently introduced method based on the geometric interpretation of linear momentum of vortex lines to determine dynamical properties of a network of knots and links. To show how the method works and to prove its feasibility, we consider the evolution of quantum vortices governed by the Gross-Pitaevskii equation. Accurate estimates of the momentum of interacting and reconnecting vortex rings, links, and knots are determined. The method is of general validity and it proves particularly useful in practical situations where no analytical information is available. It can be easily adapted to situations where morphological information can be extracted from experimental or computational data, thus providing a powerful tool for realtime diagnostics of vortex filaments or other networks of filamentary structures.
\end{abstract}

DOI: 10.1103/PhysRevE.100.011101

\section{INTRODUCTION}

The results obtained by direct application of a technique introduced recently by one of us [1,2] to determine the impulse associated with vortex motion are presented. The method is based on the geometric interpretation of linear and angular momentum of vortex filaments in terms of weighted area of projected graphs. An accurate estimate of the momentum is obtained by computing the individual momentum components from direct measurements of the area regions of planar graphs obtained by planar projections of the vortex lines. By assigning appropriate weights to each graph region one can thus compute the momentum components directly from data analysis.

The method proves particularly useful when we cannot rely on analytical description of vortex line evolution, or when dynamical information has high computational costs. In these cases direct implementation of the method provides realtime estimates of three-dimensional (3D) dynamical properties of interacting filaments under continuous reconnections and restructuring [3, 4]. Moreover, the method can be readily employed by direct analysis of two-dimensional images from laboratory experiments, as in the case of the production of defects in solid state physics [5] and vortex knots in water [6], or from natural observations, as in the case of eruption of plasma loops in the solar corona and localized, strong magnetic fields in astrophysical flows [7,8].

To illustrate how the method works we consider the evolution of reconnecting vortex links, rings, and knots governed by the Gross-Pitaevskii equation. Since the dynamics of these objects is obtained by postprocessing of direct numerical

\footnotetext{
*simone.zuccher@univr.it

†renzo.ricca@unimib.it
}

simulations, the method is particularly well suited. For this we rely on data obtained by previous numerical simulations [9 10 and new results of vortex knots evolution. The method has been already applied in some simple cases [11], but a detailed physical justification and an accurate description of its implementation is provided here. In Sec. II we briefly review how the momentum of a physical system of filaments can be related to the concept of projected area. In Sec. III we provide a physical justification of the weighted area concept, and in Sec. IV we discuss its numerical implementation. In Sec. V we apply the method to the case of interacting vortex links [10], reconnecting rings [9], and evolution of vortex knots (trefoil and cinquefoil). Final remarks on possible refinements and extensions of this method are presented in Sec. VI.

\section{LINEAR MOMENTUM IN TERMS OF PROJECTED AREA}

Let us consider the evolution of quantum vortices governed by the 3D Gross-Pitaevskii equation (GPE) [12,13], given in nondimensional form by

$$
\frac{\partial \psi}{\partial t}=\frac{i}{2} \nabla^{2} \psi+\frac{i}{2}\left(1-|\psi|^{2}\right) \psi
$$

for the complex wave function $\psi$, with background unit density $\rho=|\psi|^{2} \rightarrow 1$ as $|x| \rightarrow \infty$. It is well known [14] that by using the Madelung transformation $\psi=\sqrt{\rho} \exp (i \theta)$ the real and imaginary parts of $(1)$ give rise to a momentum and a continuity equation of a fluidlike medium of density $\rho$ and velocity $\mathbf{u}=\nabla \theta$, providing a hydrodynamic interpretation of GPE in terms of macroscopic quantities. Quantum vortices are thus zero-density lines embedded in a unit density fluid medium. Hence density is not constant everywhere. However, to a good approximation, the density profile reaches a plateau in a region of the order of a few healing lengths 
from the defect line [15]. We can thus think of a two-fluid region, one in the tubular neighborhood of a nodal line, where compressible (acoustic) effects dominate, and one outside this tubular neighborhood, essentially incompressible. Since the healing length $\xi$ of a defect is several orders of magnitude smaller than the typical length scale, we can assume that compressible effects remain localized within such a tubular region, considering this as an "excluded volume" associated with the defect. For the present purpose, however, we will omit this artificial decomposition, regarding the whole fluid region as incompressible.

During evolution GPE is known to conserve total mass, energy, and momentum [16]. From a hydrodynamic perspective quantum vortices move according to Eulerian dynamics [17-19], and are subject to long-distance interactions by the Biot-Savart law and change of topology by local reconnections. Several dynamical features can thus be inferred from a classical viewpoint, such as the linear momentum (per unit density) of a vortex [20]. For localized vorticity this can be written as

$$
\mathbf{P}=\frac{1}{2} \int_{V(\omega)} \mathbf{X} \times \omega d V
$$

where $V=V(\omega)$ is the volume of the domain of definition of vorticity and $\mathbf{X}$ the position vector. For quantum defects vorticity is localized on nodal lines $\chi$ of the wave function $\psi$; hence, we can identify $\omega$ with the unit tangent to $\chi$, with $\mathbf{X}=\mathbf{X}(s)(s$ arc-length on $\chi)[21] ;$ thus, we have

$$
\mathbf{P}=\frac{1}{2} \Gamma \oint_{\chi} \mathbf{X} \times \frac{d \mathbf{X}}{d s} d s=\frac{1}{2} \Gamma \oint_{\chi} \mathbf{X} \times d \mathbf{X},
$$

where $\Gamma=2 \pi$ is the quantized circulation. In general an analytic expression for $\mathbf{P}$ is not available. A method can be developed on the basis of a geometric interpretation of the last integrand in terms of elementary area: since $\mathbf{X} \times d \mathbf{X}=2 d \mathcal{A}$, where $d \mathcal{A}$ is an elementary area of unit normal $\hat{\mathbf{e}}_{\mathcal{A}}$, we have

$$
\mathbf{P}=\Gamma \hat{\mathbf{e}}_{P} \int_{\mathcal{A}} d \mathcal{A},
$$

where now $\mathcal{A}$ denotes the area of the region bounded by $\chi$ projected along the unit vector $\hat{\mathbf{e}}_{P}=\mathbf{P} /|\mathbf{P}|$. The vortex line(s) projected along $\hat{\mathbf{e}}_{P}$ will form a planar, oriented graph $\vec{\Lambda}$ with orientation induced by $\omega$. If defects are given by knots and links in space [as shown in Fig. 1(a)] the projected graph $\vec{\Lambda}_{i}$ along the direction of projection $\hat{\mathbf{e}}_{i}$ (where $i=x, y, z$ denotes the relative direction of a principal axis) will be made by a finite collection of regions-the faces of $\vec{\Lambda}_{i}$-denoted by $R_{j}$ $(j=1,2, \ldots, n)$. Hence, if $\mathbf{P}=\left(P_{x}, P_{y}, P_{z}\right)$, we can write

$$
P_{i}=\Gamma \hat{\mathbf{e}}_{P} \cdot \hat{\mathbf{e}}_{i} \int_{\mathcal{A}\left(\vec{\Lambda}_{i}\right)} d \mathcal{A}=\Gamma \mathcal{A}_{i}, \quad i=x, y, z,
$$

where $\mathcal{A}_{i}=\mathcal{A}_{i}\left(\Lambda_{i}\right)$ denotes the area of the region bounded by $\Lambda_{i}$. There is no difficulty to interpret $\mathcal{A}_{i}$ as standard area when the projected graph is given by a collection of simple, non-self-intersecting, plane curves. If, however, $\vec{\Lambda}_{i}$ is a graph whose diagram has self-intersections [as in Fig. 1(b)], an appropriate definition of area is needed.

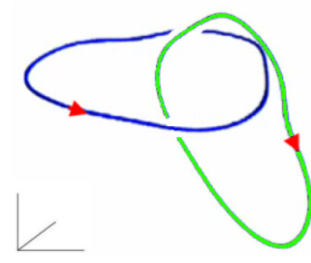

(a)

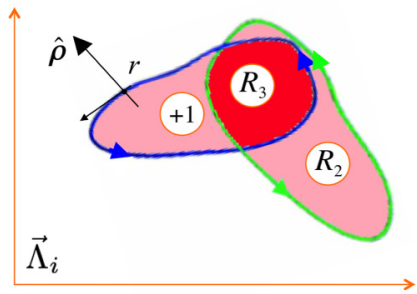

(c)

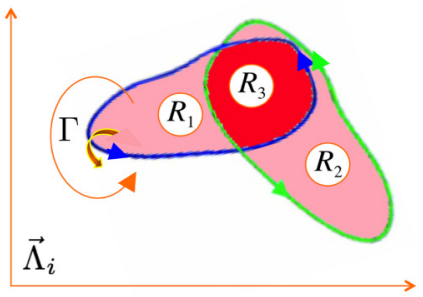

(b)

(d)

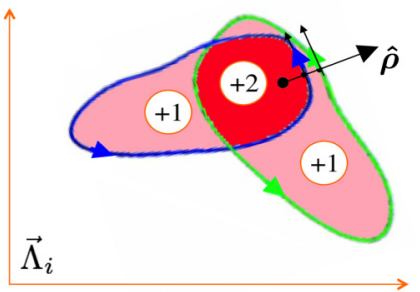

FIG. 1. (a) Two defects of same circulation $\Gamma=2 \pi$ forming a Hopf link in space (arrows denote vorticity direction). (b) Graph $\vec{\Lambda}_{i}$ obtained by the planar projection of the Hopf link along the direction $\hat{\mathbf{e}}_{i}$ : each region, $R_{1}, R_{2}$, and $R_{3}$, is pierced by the streamlines due to the circulation of all vortex strands. (c) The index $\mathcal{I}_{j}=\mathcal{I}_{j}\left(R_{j}\right)$ $(j=1,2,3)$ is given by the algebraic sum of the signed intersections $\hat{\rho} \cap \vec{\partial} R_{j}$. (d) $\mathcal{I}_{1}\left(R_{1}\right)=+1, \mathcal{I}_{2}\left(R_{2}\right)=+1, \mathcal{I}_{3}\left(R_{3}\right)=+2$.

\section{WEIGHTED AREA OF ORIENTED GRAPH}

From a physical viewpoint, Eq. (5) relates the momentum component $P_{i}$ to the flux of circulation through $\mathcal{A}_{i}$. The streamlines associated with such flux pierce each face of the graph $\vec{\Lambda}_{i}$. Since each region $R_{j}$ is bounded by an oriented boundary $\vec{\partial} R_{j}$ made by arcs carrying their own circulation, each face will thus contribute to the momentum with area and flux determined by multiplying the standard area $A_{j}=A_{j}\left(R_{j}\right)$ by the total flow of circulation given by the neighboring arcs [note that these in general need not be congruently oriented; see Fig. 1(b)]. Since any point of $R_{j}$ is subject to the same total flux, the sum of the individual circulations will contribute to an index $\mathcal{I}_{j}=\mathcal{I}_{j}\left(R_{j}\right)$ that is a topological invariant of $R_{j}$. This can indeed be identified with the Gauss linking number of the streamlines with the oriented boundary $\vec{\partial} R_{j}$. This index is the intersection number of $R_{j}$ [22,23], and it is given by

$$
\mathcal{I}_{j}=\sum_{r \in\left\{\hat{\rho} \cap \vec{\partial} R_{j}\right\}} \epsilon_{r},
$$

where $\epsilon_{r}= \pm 1$ denotes the algebraic sign of each intersection $r \in\left\{\hat{\boldsymbol{\rho}} \cap \vec{\partial} R_{j}\right\}$ determined by the following procedure: (i) from an arbitrarily chosen point of $R_{j}$ draw a director line in any arbitrary direction $\hat{\rho}$; (ii) determine the intersection(s) $r \in$ $\left\{\hat{\boldsymbol{\rho}} \cap \vec{\Lambda}_{i}\right\}$ of this line with $\vec{\Lambda}_{i}$ and consider the base pair $\{\hat{\boldsymbol{\rho}}, \hat{\mathbf{t}}\}$ at each $r$ (t) being the local unit tangent to $\vec{\partial} R_{j}$ ); (iii) assign to each intersection the value \pm 1 according to the right-hand rule. The algebraic sum of all \pm 1 's thus obtained for each $R_{j}$ is the index $\mathcal{I}_{j}\left(R_{j}\right)$. We can then define the weighted area 

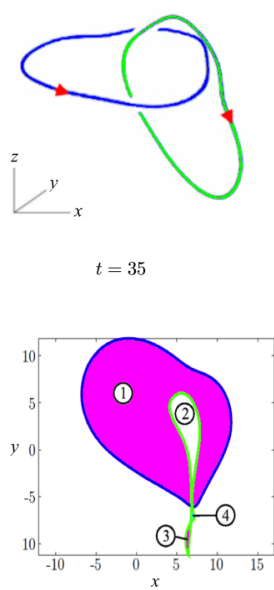

\begin{tabular}{|c|c|c|c|c|c|c|c|c|}
\hline \multicolumn{3}{|c|}{$x-y$ plane } & \multicolumn{3}{|c|}{$z-x$ plane } & \multicolumn{3}{|c|}{$y-z$ plane } \\
\hline regi & on area & index & regio & area & index & regior & area & index \\
\hline$R_{1}$ & 201.35 & +1 & $\mathrm{R}_{1}$ & 13.26 & +1 & $\mathrm{R}_{1}$ & 199.48 & +1 \\
\hline $\mathrm{R}_{2}$ & 18.14 & 0 & $\mathrm{R}_{2}$ & 13.93 & -1 & $\mathrm{R}_{2}$ & 22.85 & 0 \\
\hline $\mathrm{R}_{3}$ & 0.94 & +1 & $\mathrm{R}_{3}$ & 3.78 & 0 & $\mathrm{R}_{3}$ & 0.34 & -1 \\
\hline $\mathrm{R}_{4}$ & 0.39 & -1 & $\mathrm{R}_{4}$ & 0.83 & -1 & $\mathrm{R}_{4}$ & 0.86 & +1 \\
\hline & & & $\mathrm{R}_{5}$ & 1.70 & +1 & $\mathrm{R}_{5}$ & 0.00 & -1 \\
\hline+1 & \begin{tabular}{l|l}
0 & -1
\end{tabular} & & $\mathrm{R}_{6}$ & 3.15 & +1 & & & \\
\hline & & & $\mathrm{R}_{7}$ & 2.18 & -1 & & & \\
\hline
\end{tabular}
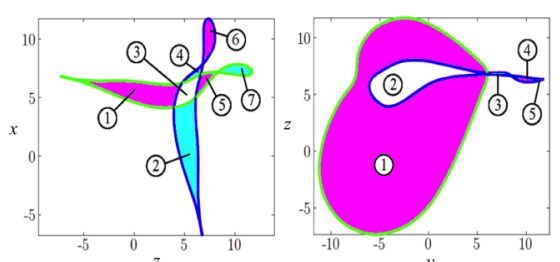
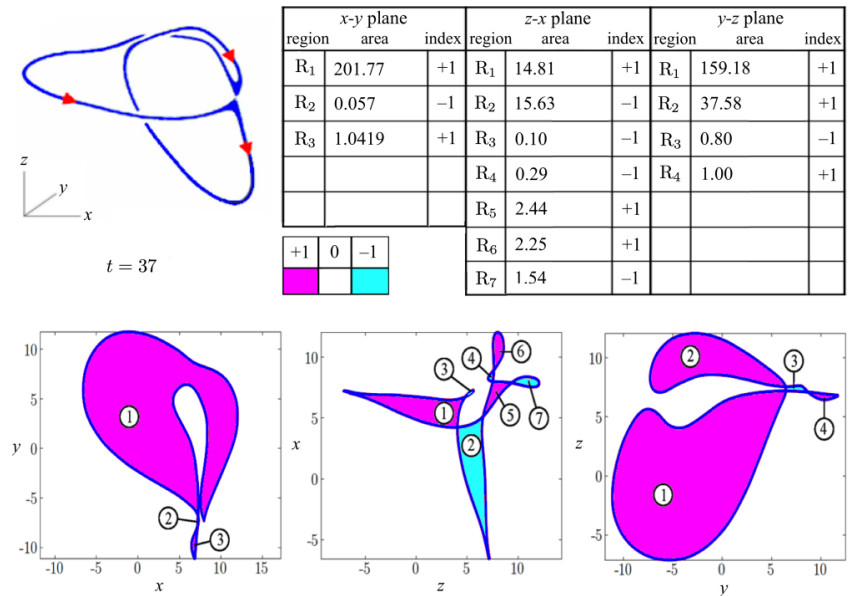

FIG. 2. Application of the geometric method to the evolution of Hopf link defects, just before $(t=35)$ and after $(t=37)$ a reconnection event. Top row: (left) 3D configuration of defects in space; (right) numerical values of standard area and index of the individual graph regions identified in the projection planes $z=0, y=0, x=0$. Bottom row: graph regions color-coded (online) according to the values of $\mathcal{I}_{j}=0, \pm 1$ [magenta (dark gray): $\mathcal{I}=+1$; white: $\mathcal{I}=0$; cyan (light gray): $\mathcal{I}=-1$ ].

$\mathcal{A}_{i}=\mathcal{A}\left(\vec{\Lambda}_{i}\right)$ by

$$
\mathcal{A}_{i}=\sum_{j} \mathcal{I}_{j} A_{j i}
$$

where $A_{j i}=A_{j i}\left(R_{j}\right)$ denotes the standard area of $R_{j}$.

For a tangle of $N$ knots and links of different circulations $\Gamma_{k}(k=1, \ldots, N)$ we can then extend the above result straightforwardly. Thus, we have the following.

Theorem 1. The linear momentum components $P_{i}$ of a tangle of $N$ vortices of circulation $\Gamma_{k}(k=1, \ldots, N)$ can be written in terms of weighted areas by

$$
P_{i}=\sum_{j} \bar{\Gamma}_{j} A_{j i}, \quad \text { with } \quad \bar{\Gamma}_{j}=\sum_{r} \epsilon_{r} \Gamma_{r, k}
$$

where $\bar{\Gamma}_{j} A_{j i}$ denotes the weighted circulation of the standard area $A_{j i}$ of $R_{j}$, and $\Gamma_{r, k}$ the circulation $\Gamma_{k}$ at each intersection site $r \in\left\{\hat{\boldsymbol{\rho}} \cap \vec{\Lambda}_{i}\right\}$.

\section{IMPLEMENTATION OF NUMERICAL DIAGNOSTICS}

Given the numerical solution $\psi$ at each grid point in space and time, the first step consists of retrieving the vortex centerlines. Instead of using previous methods [9]10], we found it computationally convenient to exploit the fact that the GPE isophases are Seifert surfaces of the vortex lines [24]. These latter can be obtained by extracting the boundary points from the arbitrary isophase surface computed numerically. This approach reduces considerably the computational cost of data extraction. Since the vortex lines are given computationally by polygonal curves, the number of nodal points in projected graphs increases quadratically with the number of polygonal segments [25]. Loops relatively dense in space require a finer discretization, something that can be achieved by automated diagnostics. The next step is to obtain "good projections": this is done by ensuring that all nodal points in the projected graph are double points, i.e., points of intersection given by at most two self-intersecting arcs. If a nodal point has degree $m>2$, it can be split into $m$ double points by numerical perturbation of the projection map, so as to ensure a required goodness of the graph.

The most difficult task is represented by the numerical identification of the individual loops present in the planar projection (see, e.g., the diagrams of Fig. 2) and the correct assignment of the arcs to the appropriate vortex line. If the coordinates of each node are not difficult to be determined, the reconstruction of the individual loops and the correct identification of the appropriate areas and weights require some careful analysis. This is a delicate, crucial step of the procedure, so any local grid refinement should be aimed at optimizing this task in particular. An appropriate algorithm has thus been developed and implemented for complex configurations made, however, of a quite limited number of closed loops. Application to highly complex networks of filaments has not been done and at this stage of things it might be extremely time-consuming.

\section{MOMENTUM OF RECONNECTING LINKS, RINGS, AND KNOTS}

The numerical scheme has been applied to determine the linear momentum associated with the evolution of (i) the Hopf link of Fig. 1(a), (ii) a pair of interacting and reconnecting rings (Fig. 4), and (iii) two vortex knot types (Fig. 5), all with same circulation $\Gamma=2 \pi$.

Hopf link. At $t=0$ the two rings both have radius $R=8$, and are placed on mutually orthogonal planes centered at $(0.5,4.5,0)$ in the plane $z=0$, and at $(0,-4,0)$ in the plane $x=0$. The computational domain is given by $[-20 ; 20] \times$ $[-25 ; 25] \times[-20 ; 30]$ with the same spatial discretization in each direction $\Delta=\xi / 3$, and time step 0.0125 units. For illustration purposes we show (Fig. 2) details of the application of the method at two instances, immediately before and after a reconnection event. For this we rely on data extracted from the simulation studied in [10]. For each time step we show (top) the space configuration of defects and the values of standard area and index of the individual graph regions identified in each projection plane $z=0, y=0, x=0$, and 

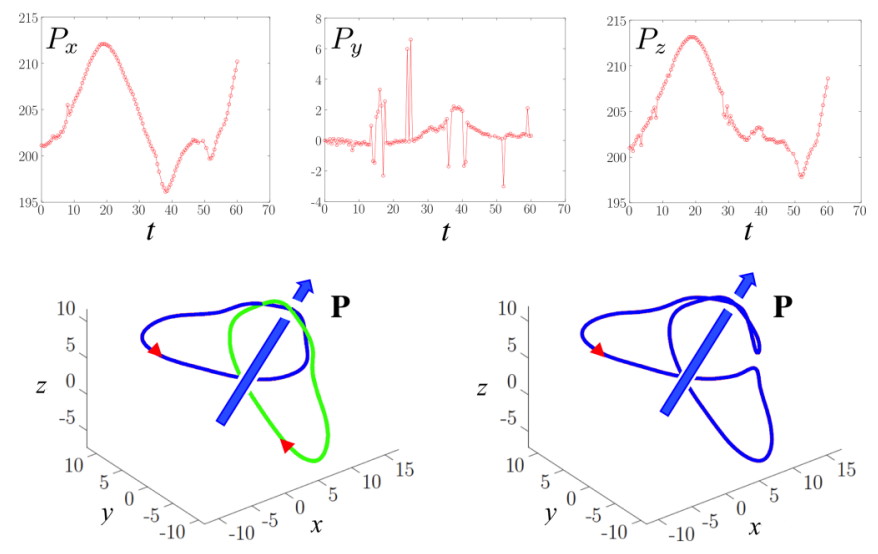

$$
t=35
$$$$
t=37
$$

FIG. 3. Top row: plots of the momentum components $P_{x}, P_{y}$, and $P_{z}$ against time $t$. Bottom row: direction and intensity of the linear momentum [big arrow (blue online)] just before and after the reconnection event.

(bottom) the relative color-coded graphs. Areas with index $\mathcal{I}=+1$ (color-coded online by magenta) indicate contribution to the relative momentum component along the positive axis direction, whereas areas with index $\mathcal{I}=0$ (color-coded by white) provide no contribution. Note that small areas with large index may contribute to the momentum more than large areas with small index, consistently with physical intuition. The algebraic index attributed to given areas may thus considerably influence the resulting momentum. In particular, dominant contributions from negative indices may even reverse motion [26]. Direct implementation of this method allows one to determine time-dependent information of each momentum component, which is useful for assessing relative contributions (as shown in the top diagrams of Fig. 3 , where $\left|P_{y}\right|$ results are negligible with respect to the other two components). Real-time visualization of direction and intensity of total momentum is shown by the big arrow in the two diagrams at the bottom of Fig. 3 .

Planar rings. Figure 4 shows the interaction and reconnection of two planar rings as studied in [9]. At time $t=0$
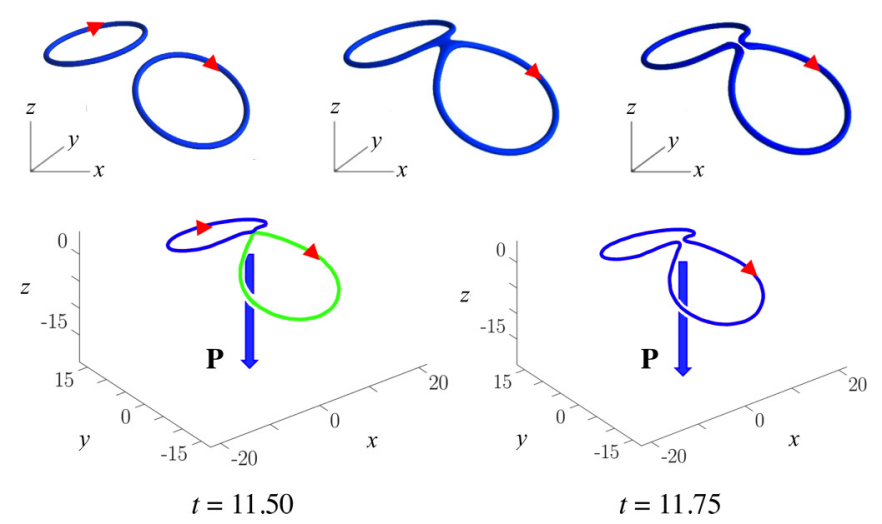

FIG. 4. Top row: interaction and reconnection of two planar rings (from [9]). Bottom row: direction and intensity of the linear momentum [big arrow (blue online)] just before and after the reconnection event.
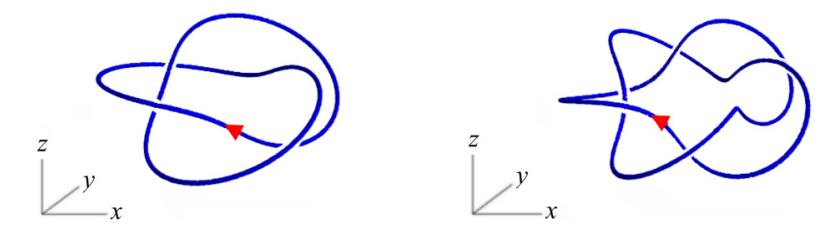

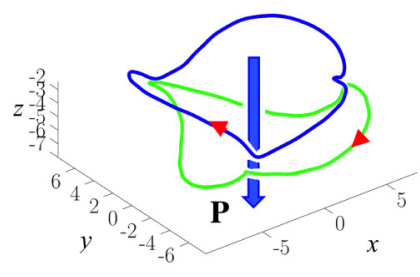

(a)

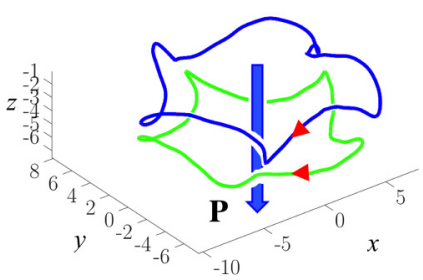

(b)
FIG. 5. Top row: initial configuration of (a) trefoil knot $\mathcal{T}_{2,3}$ and (b) cinquefoil $\mathcal{T}_{2,5}$. Bottom row: direction and intensity of linear momentum shown by the big arrow (blue online) immediately after simultaneous reconnections.

the two rings are initially unlinked, centered at $(0 ; \pm 10 ; 0)$, with radius $R=8$ and mutually inclined by an angle $\alpha=$ $\pm \pi / 10$ with respect to the plane $z=0$. Since the initial condition is highly symmetric, the momentum remains essentially oriented along a single direction. Particularly interesting is the case of the head-on collision of two parallel, coaxial vortex rings (not studied here), where upon interaction and subsequent reconnections a regular crown of ringlets is formed [27,28]. Since the system is perfectly axially symmetric, crude estimates based on weighted area information have allowed one to predict [2] qualitative and quantitative features, such as the production of oppositely directed ringlets through the redistribution of (zero) total momentum and the follow-up dynamics immediately after the reconnection stage.

Torus knots. Figure 5 shows two torus knots $\mathcal{T}_{p, q}$ given by the parametric equations

$$
\mathbf{X}:\left\{\begin{array}{l}
x(t)=\cos (p t)[R+r \cos (q t)], \\
y(t)=\sin (p t)[R+r \cos (q t)], \\
z(t)=r \sin (q t),
\end{array}\right.
$$

where $p$ and $q$ denote, respectively, the number of wraps along the longitudinal and meridian direction of the torus of large radius $R$ and small radius $r$. The initial condition is prescribed as follows. Given $p, q, R$, and $r$, for each point $P$ in the numerical domain we look for the closest point $Q \in \mathcal{T}_{p, q}$ so that $\overrightarrow{Q P}$ is in the cross-sectional plane normal to the local tangent to $\mathcal{T}_{p, q}$ at $Q$. By taking $(d, \theta)$ the polar coordinates of $P$ with respect to $Q$, where $d=|\overrightarrow{Q P}|$ and $\theta$ the angle between the unit principal normal and $\overrightarrow{Q P}$, the initial condition is given by $\psi_{0}=\sqrt{\rho_{0}} \exp \left(i \theta_{0}\right)$, where $\rho_{0}$ is given by a new, higher-order Padé approximation (see [29] for details). The computational domain is set to $[-20 ; 20] \times[-20 ; 20] \times$ $[-25 ; 15]$, with number of grid points $150^{3}$, spatial resolution $\Delta=(4 / 15) \xi$, and time step 0.0125 units. At $t=0$ the torus knot is centered at $x=y=z=0$. For both knots $\mathcal{T}_{2,3}$ and $\mathcal{T}_{2,5}$ we take $R=6$ and $r=2$. Since during evolution the initial symmetry is preserved, the direction of the total momentum 
remains unchanged before and after reconnection, as shown by the big arrow in the plots of Fig. 5 (bottom row).

\section{CONCLUDING REMARKS}

The geometric method presented provides real-time estimates of the linear momentum and represents a useful tool for diagnostics and predictive analysis of dynamical aspects of networks of filamentary structures, especially when no analytical information is available. The examples given here are based on data analysis of the evolution of GPE defects, and they serve the purpose to illustrate how the method can be applied to complex systems. The relationship between linear momentum and weighted area, upon which the method relies, is exact in the limit of thin filaments in incompressible fluid.

The method could also provide useful information in the compressible case too, where, as in the GPE case, one can use it to compare incompressible information with known conservation of momentum to obtain acoustic emission estimates. In such cases, since compressible effects remain localized in defect regions, we conjecture that vortex lines could be artificially endowed with virtual tubes (by making use of Padé approximation), whose volume would play a similar role of the excluded volume of polymers. These tubular regions could then be removed from the projected areas, so as to have more accurate estimates from the incompressible part. We believe this technique could be easily implemented in future work. Another matter is direct comparison with conservation of momentum in GPE. This is a very delicate issue, since we know that various factors may influence the result, one for all the relative size of the defects compared to the size of the numerical box. This issue is probably resolved by very high computational power.

The methodology used here, in any case, proves robust and can be equally applied to networks of filamentary structures in superfluid, classical, or magnetic systems, where localized fields are present. Data can be obtained from laboratory experiments as well as numerical simulations. In the former case data extraction and processing may vary according to context. In the case of vortex filaments in water or in superfluid helium, the reconstruction of vortex morphology can be achieved by collecting volumetric data from laser illuminated slices of vortex core regions and then by postprocessing the raw data by fast-marching algorithms for tracing the vortex lines in space. This technique, which was successfully employed for the postprocessing analysis of vortex knots in water [6] and in superfluid helium [30], can be readily implemented for our purpose. Other techniques based on photoemission spectroscopy measurements (for defects in crystals), electron microscopy and sound propagation (for vortices in superconductors and condensates), or filtered image analysis (for coronal loops in solar physics) can well provide equally similar information. More obvious is the implementation of our method in direct numerical simulations of complex vortex tangles in fluid flows (classical or superfluid), where one new route to explore may be represented by the use of quantum trajectory methods in the hydrodynamical formulation of quantum dynamics [31].
[1] R. L. Ricca, Momenta of a vortex tangle by structural complexity analysis, Physica D: Nonlinear Phenomena 237, 2223 (2008).

[2] R. L. Ricca, Impulse of vortex knots from diagram projections, in Topological Fluid Dynamics: Theory and Applications, edited by H. K. Moffatt et al. (Elsevier, New York, 2013) [Procedia IUTAM 7, 21 (2013)].

[3] C. F. Barenghi, R. L. Ricca, and D. C. Samuels, How tangled is a tangle, Physica D (Amsterdam, Neth.) 157, 197 (2001).

[4] A. Villois, D. Proment, and G. Krstulovic, Evolution of a superfluid vortex filament tangle driven by the Gross-Pitaevskii equation, Phys. Rev. E 93, 061103(R) (2016).

[5] S. Lugomer, Observation of laser-induced microscale knotted and unknotted vortex filaments on vaporizing tantalum surface, Phys. Rev. B 54, 4488 (1996).

[6] D. Kleckner and W. T. M. Irvine, Creation and dynamics of knotted vortices, Nat. Phys. 9, 253 (2013).

[7] A. L. Wilmot-Smith, D. I. Pontin, and G. Hornig, Dynamics of braided coronal loops I. Onset of magnetic reconnection, Astron. Astrophys. 516, A5 (2010).

[8] T. Amari, A. Canou, J.-J. Aly, F. Delyon, and F. Alauzet, Magnetic cage and rope as the key for solar eruptions, Nature (London) 554, 211 (2018).

[9] S. Zuccher and R. L. Ricca, Helicity conservation under quantum reconnection of vortex rings, Phy. Rev. E 92, 061001 (2015).
[10] S. Zuccher and R. L. Ricca, Relaxation of twist helicity in the cascade process of linked quantum vortices, Phy. Rev. E 95 , 053109 (2017).

[11] F. Maucher, S. A. Gardiner, and I. G. Hughes, Excitation of knotted vortex lines in matter waves, New J. Phys. 18, 063016 (2016).

[12] E. P. Gross, Hydrodynamics of a superfluid condensate, J. Math. Phys. 4, 195 (1963).

[13] L. P. Pitaevskii, Vortex lines in an imperfect Bose gas, J. Exptl. Theoret. Phys. (U.S.S.R.) 40, 646(1961) [Sov. Phys. JETP 13, 451 (1961)].

[14] C. F. Barenghi and N. G Parker, A Primer on Quantum Fluids (Springer-Verlag, Berlin, 2016).

[15] S. Zuccher, M. Caliari, A. W. Baggaley, and C. F. Barenghi, Quantum vortex reconnections, Phys. Fluids 24, 125108 (2012).

[16] C. Nore, M. Abid, and M. E. Brachet, Decaying Kolmogorov turbulence in a model of superflow, Phys. Fluids 9, 2644 (1997).

[17] L. Fetter, Vortices in an imperfect Bose gas IV. Translational velocity, Phys. Rev. 151, 100 (1966).

[18] C. Neu, Vortices in complex scalar fields, Physica D 43, 385 (1990).

[19] F. Lund, Defect dynamics for the nonlinear Schrödinger equation derived from a variational principle, Phys. Rev. Lett. A 159. 245 (1991). 
[20] G. Batchelor, An Introduction to Fluid Dynamics (Cambridge University Press, Cambridge, 1967).

[21] P. G. Saffman, Vortex Dynamics (Cambridge University Press, Cambridge, 1991).

[22] D. Rolfsen, Knots and Links (AMS Chelsea Publishing, Providence, RI, 1990).

[23] R. L. Ricca and B. Nipoti, Gauss linking number revisited, J. Knot Theory Ramifications 20, 1325 (2011).

[24] S. Zuccher and R. L. Ricca, Twist effects in quantum vortices and phase defects, Fluid Dyn. Res. 50, 011414 (2018).

[25] R. L. Ricca, On simple energy complexity relations for filament tangles and networks, Complex Systems 20, 195 (2012).

[26] C. F. Barenghi, R. Hanninen, and M. Tsubota, Anomalous translational velocity of vortex ring with finite amplitude Kelvin waves, Phys. Rev. E 74, 046303 (2006).
[27] T. T. Lim, and T. B. Nickels, Instability and reconnection in the head-on collision of two vortex rings, Nature (London) 357, 225 (1992).

[28] M. Cheng, J. Lou, and T. T. Lim, Numerical simulation of headon collision of two coaxial vortex rings, Fluid Dyn. Res. 50, 065513 (2018).

[29] M. Caliari and S. Zuccher, Reliability of the time splitting Fourier method for singular solutions in quantum fluids, Comput. Phys. Commun. 222, 46 (2018).

[30] G. P. Bewley, M. S. Paoletti, K. R. Sreenivasan, and D. P. Lathrop, Characterization of reconnecting vortices in superfluid helium, Proc. Natl. Acad. Sci. USA 105, 13707 (2008).

[31] R. E. Wyatt, Quantum Dynamics with Trajectories. Introduction to Quantum Hydrodynamics (Springer, New York, 2005). 